\title{
Neonatal Development of the Corneal Stroma in Wild-Type and Lumican-Null Mice
}

\author{
Nicola Beecher, ${ }^{1}$ Shukti Chakravarti, ${ }^{2}$ Sarab Joyce, ${ }^{2}$ Keith M. Meek, ${ }^{1}$ and \\ Andrew J. Quantock ${ }^{1}$
}

Purpose. Between days 8 and 14 of neonatal development, the corneal stroma of the mouse undergoes critical changes in tissue thickness, cell density, and light scattering. The authors investigate the stromal matrix structure in wild-type and lumican-deficient corneas in this developmental phase.

Methods. Wild-type $(n=44)$ and lumican-deficient $(n=42)$ mouse corneas at neonatal days $8,10,12$, and 14 were investigated by synchrotron $\mathrm{x}$-ray diffraction to establish the average collagen fibril spacing, average collagen fibril diameter, and level of fibrillar organization in the stromal matrix.

Results. Collagen interfibrillar spacing in the normal mouse cornea became more closely packed between days 8 and 14, though not significantly so. In lumican-null mice, interfibrillar spacing was significantly elevated at days 8,10 , and 12 , but not day 14, compared with that in wild-type mice. At all stages investigated, collagen fibrils were, on average, marginally thinner than normal in lumican-null mutants, and the spatial distribution of the fibrils was less well organized.

Concuusions. Transient thickening of the corneal stroma of the normal mouse at eye opening is probably not caused by widespread, homogeneous rearrangement of collagen fibrils but more likely by a temporary increase in cell or stromal "lake" volume. Lumican, structurally influential in adult mouse corneas, is also a key molecule in the neonatal development of the stromal matrix. (Invest Ophthalmol Vis Sci. 2006;47:146-150) DOI:10.1167/iovs.05-0907

$\mathrm{T} \mathrm{n}$ the corneal stroma, collagen fibrils are uniformly thin, regularly spaced, and aligned with high spatial order. This arrangement is necessary for light transmittance, ${ }^{1}$ with proteoglycans in the extracellular stromal matrix implicated in the control of the collagen fibrillar architecture. The role of proteoglycans in the governance of corneal structure has long been a matter of interest. For example, after the discovery by Anseth $^{2}$ that the wound area and the surrounding tissue of

From the ${ }^{1}$ Structural Biophysics Group, Cardiff School of Optometry and Vision Sciences, Cardiff University, Cardiff, United Kingdom; and the ${ }^{2}$ Departments of Medicine, Cell Biology and Ophthalmology, Johns Hopkins University, Baltimore, Maryland.

Supported by the Biotechnology and Biological Sciences Research Council Project Grant 72/B18021 (AJQ), the National Institutes of Health Grant EY11654 (SC), the Medical Research Council Programme Grant G0001033 (KMM, AJQ), and the Council for the Central Laboratory of the Research Councils for beam time at the Synchotron Radiation Source (SRS), Daresbury Laboratory, Cheshire, UK (KMM, AJQ).

Submitted for publication July 14, 2005; revised August 19, 2005; accepted November 15, 2005.

Disclosure: N. Beecher, None; S. Chakravarti, None; S. Joyce, None; K.M. Meek, None; A.J. Quantock, None

The publication costs of this article were defrayed in part by page charge payment. This article must therefore be marked "advertisement" in accordance with 18 U.S.C. $\$ 1734$ solely to indicate this fact.

Corresponding author: Andrew J. Quantock, Structural Biophysics Group, Cardiff School of Optometry and Vision Sciences, Cardiff University, Redwood Building, King Edward VII Avenue, Cathays Park, Cardiff CF10 3NB, UK; quantockaj@cf.ac.uk. rabbit corneas have high levels of chondroitin sulfate and dermatan sulfate and low levels of keratan sulfate, Hassell and colleagues $^{3}$ reported that opaque corneal scar tissue in rabbits contains negligible amounts of keratan sulfate and displays large interfibrillar spaces. A subsequent increase in keratan sulfate is accompanied by a return to normal fibril spacing. A significant reduction in keratan sulfate levels in the cornea is also often seen in patients with the most common form of macular corneal dystrophy, ${ }^{4,5}$ a disease that renders the cornea significantly thinner than normal with more closely packed collagen fibrils. ${ }^{6}$ In development, too, keratan sulfate has long been linked with stromal ultrastructure, ${ }^{7}$ and the appearance of the sulfated proteoglycan form of lumican after embryonic day 12 in the chick cornea has given rise to the idea that it might be associated with changes in corneal structure, though other regulatory mechanisms are likely involved. ${ }^{8,9}$

Three types of small corneal proteoglycan have been identified that contain the keratan sulfate side chains lumican, ${ }^{10}$ keratocan, ${ }^{11}$ and mimecan. ${ }^{12}$ Advances in the field of molecular genetics have allowed the generation of mice with targeted proteoglycan deficiencies, allowing investigations into the functional roles of specific corneal proteoglycans. This work has shown that, in maturity, neither keratocan-deficient nor mimecan-deficient corneas exhibit obvious corneal opacities. ${ }^{13,14}$ The adult lumican-deficient mouse, on the other hand, displays a severe phenotype, with homozygous mutants exhibiting pronounced corneal opacification, especially in deeper stromal layers. ${ }^{15}$ What is not known in any detail, however, is whether ultrastructural matrix aberrations caused by the lack of lumican are late-evolving phenomena or early events in neonatal development that persist into adulthood.

The mouse cornea is well formed at birth but undergoes significant growth afterward, characterized by changes in tissue thickness, cell density, and light scattering. ${ }^{16}$ The highly organized stromal matrix, centrally important for light transmittance, clearly must adjust structurally during this developmental phase, but little is known about the manner in which it does so. The mouse as an animal model for corneal research is increasingly gaining importance because of the availability of gene-targeted mutants, and systematic examination of the collagen architecture in the mouse cornea by transmission electron microscopy has begun to address the important question of postnatal development with a view to understanding the acquisition of corneal transparency. This will provide a good assessment of collagen fibril ultrastructure in ultrathin tissue sections. We can also carry out $\mathrm{x}$-ray scattering experiments on intact, isolated corneas to ascertain the spatial dimensions of fibrillar collagen in the corneal stroma. In recent years we have used this approach to study the mature corneas of normal mice and of mice with null mutations in lumican, keratocan, or mimecan. ${ }^{17-19}$ Synchrotron $\mathrm{x}$-ray fiber diffraction is a technique by which an intense beam of monochromatic $\mathrm{x}$-rays, focused in the current series of experiments to $1 \mathrm{~mm} \times 0.5$ $\mathrm{mm}$ at the specimen, is passed through the whole thickness of a cornea. As with the passage of light through the cornea, each collagen fibril in the path of the x-ray beam acts as an independent scatterer of x-rays. Fiber diffraction patterns of com- 
bined x-ray scatter are recorded from individual corneas and analyzed to provide highly representative average values for the spatial dimensions of collagen fibrils in the corneal stroma. ${ }^{20}$ Our studies on 2- to 6-month-old corneas disclosed structural matrix changes in lumican-null and keratocan-null mutants but not, to any great extent, mimecan-null mutants. ${ }^{17-19}$

Recently, it was discovered that during neonatal development the mouse corneal stroma sustains a transient alteration in its physical thickness between days 8 and $14 .^{16}$ This does not happen in the corneas of lumican-null neonates. Here, we use synchrotron $\mathrm{x}$-ray fiber diffraction to investigate matrix architecture during neonatal development in wild-type and lumican-deficient corneas throughout the day 8 to 14 developmental phase. Our study provides new insights into how the collagen fibril diameter, interfibrillar spacing, and structural order adjust in the normal mouse cornea as it grows, thickens, and condenses before and after eyelid opening. Furthermore, in seeking to elucidate whether the structural defects reported in the adult lumican-null mouse cornea are caused by poor regulation of the dynamic fibrillar architecture during neonatal maturation, we provide clear evidence for a developmental role for lumican in establishing and maintaining the corneal matrix architecture.

\section{Materials ANd Methods}

\section{Specimens}

Normal mouse (CD-1) corneas at neonatal development day $8(n=10)$, day $10(n=10)$, day $12(n=12)$, and day $14(n=12)$, along with lumican-null corneas at day $8(n=12)$, day $10(n=12)$, day $12(n=$ $12)$, and day $14(n=12)$ were carefully dissected at the limbus. Because of the small size of each cornea, we thought it was more appropriate to use chemical fixation as a method of preservation rather than freezing as has been done previously, so each cornea was immediately immersed in $4 \%$ paraformaldehyde and shipped to Cardiff University in the United Kingdom. The mice were from a colony with a null homozygous mutation for lumican. ${ }^{15}$ On arrival, the corneas remained in the fixative solution at $4{ }^{\circ} \mathrm{C}$ for a week until low-angle $\mathrm{x}$-ray diffraction analysis was undertaken. All procedures were carried out in accordance with the ARVO Statement for the Use of Animals in Ophthalmic and Vision Research.

\section{Data Collection}

The corneas, secured in sealed specimen holders between two sheets of mylar, were analyzed at the Synchotron Radiation Source (SRS; Daresbury Laboratory, Cheshire, UK). Each specimen was placed in the path of a focused $(1 \times 0.5 \mathrm{~mm})$, monochromatic $(\lambda=0.154 \mathrm{~nm}) \mathrm{x}$-ray beam on SRS Station 2.1, and the shutters were opened to expose the cornea for 2 minutes. Low-angle x-ray scattering patterns for all specimens were recorded on a multiwire, gas-proportional area detector situated directly behind the cornea at a distance of $8.25 \mathrm{~m}$. An evacuated tube with polyester film windows separated the specimen from the detector to reduce air scatter. The window nearest the detector contained a lead beam stop that purposely blocked the direct x-ray beam that passed through the cornea undeviated.

\section{Data Processing}

X-ray patterns $(512 \times 512$ pixels $)$ were analyzed with software, graphics, and statistics packages (Unix-based software; Statistica; Statsoft, Tulsa, OK). They were initially normalized using ion chamber counts to account for beam intensity decay. A detector response from a 9-hour exposure to a radioactive source $\left(\mathrm{Fe}^{55}\right)$ was then subtracted from each $\mathrm{x}$-ray pattern to correct for any nonlinearities in the detector. Next, a vertical scan, 26 pixels wide, of x-ray intensity ( $I$ ) versus reciprocal space coordinate $(R)$ was taken across the center of the pattern to obtain the intensity profile of the first-order equatorial $x$-ray reflection corrected for the fact that the scan across the circular $\mathrm{x}$-ray pattern was linear. Patterns were then analyzed to obtain values for the average collagen interfibrillar spacing and average collagen fibril diameter, as described elsewhere. ${ }^{17}$ Various methods of subtracting background $\mathrm{x}$-ray scatter were tried, and after all of them the overall pattern of structural change that we report remained essentially the same.

In addition to providing a measure of collagen fibril separation, the interfibrillar reflection provides an indication of the degree of local order in the arrangement of the collagen fibrils in the cornea, based on the angular width of the interfibrillar reflection. In accordance with Stokes $^{21}$ and Regini et al., ${ }^{22}$ we represent this as the coherence distance with higher values reflective of more local order in the fibrillar array.

\section{Results}

X-ray intensity profiles from wild-type and lumican-null corneas in the neonatal day 8 to day 14 time frame contain intensity maxima representing the collagen interfibrillar $\mathrm{x}$-ray reflection (Fig. 1). Before eye opening (days 8 and 10), these maxima are generally better defined in the intensity profiles from wild-type corneas than from lumican-null corneas. This indicates that the fibrillar matrix is relatively well ordered in the normal mouse cornea throughout the developmental period studied-that is, before and after eye opening. Analysis of all x-ray patterns from the 92 corneas examined was undertaken to ascertain average collagen fibril spacing, average collagen fibril diameter, and extent of local order in the fibrillar array.

\section{Collagen Fibril Spacing}

The average center-to-center collagen interfibrillar Bragg spacing in the mouse corneal stroma did not change appreciably between postnatal days 8 and 10; it remained approximately 65 $\mathrm{nm}(P=0.953$; Table 1$)$. After eye opening, at days 12 and 14 , collagen fibril spacing was marginally lower than it was before eye opening, measuring 61 to $63 \mathrm{~nm}$ (Table 1), though this difference was not statistically significant (days 8-12, $P=$ 0.414 ; days 8-14, $P=0.063$ ). At days 8,10 , and 12, lumicandeficient corneas had significantly higher fibril spacings than their wild-type counterparts (Table 1). Later, however, at day 14 , when the average collagen fibril spacing in the lumican-null corneas decreased, no difference in collagen fibril spacing was detected between wild-type and mutant corneas (Table 1).

\section{Collagen Fibril Diameter}

In the normal mouse cornea, average collagen fibril diameters during the stated developmental period were in the $31-\mathrm{nm}$ to 32.5-nm range (Table 1 ). The average collagen fibril diameter in the same period in the lumican-null corneas was in the 29.1-nm to 30.8-nm range, lower than that in wild-types mice, with the difference significant or close to significance at all time points investigated (Table 1).

\section{Coherence Distance}

As an average value for all corneas examined at days 10, 12, and 14 , the coherence distance of the collagen fibrillar array in the corneas of lumican-null mice was low with respect to the corresponding value in the corneas of wild-type counterparts (Table 1). This points to a stromal matrix in the mouse cornea at days 10 to 14 that was less well ordered when lumican was absent, with short-range order extending approximately 30 to $50 \mathrm{~nm}$ less. In the current analyses, we were unable to reliably obtain measurements of the coherence distance from day 8 lumican-null corneas because many of the interfibrillar reflections were too diffuse. This was indicative of lower levels of structural short-range order at this time point in the mutant corneas compared to later times in these corneas. 

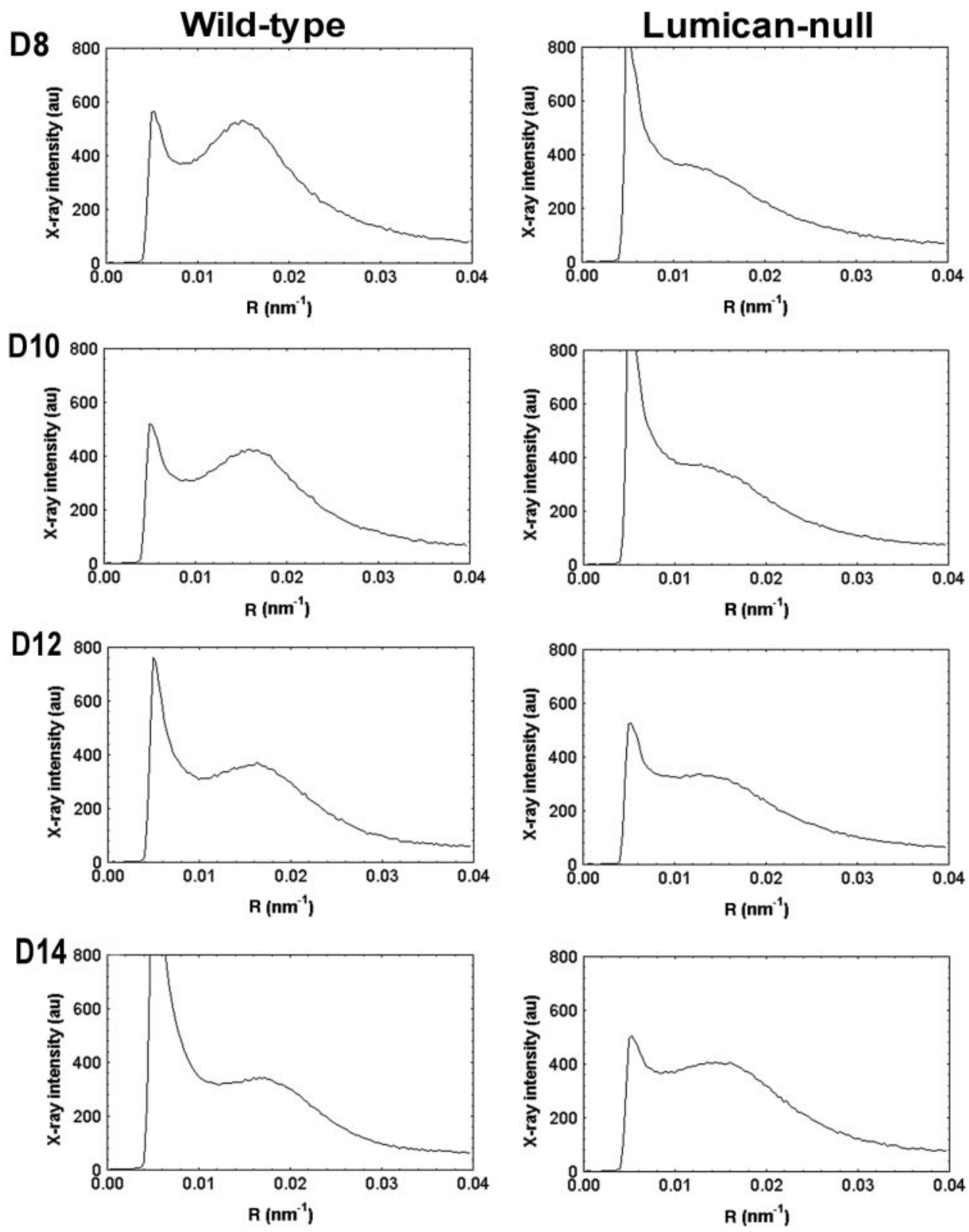

FIGURE 1. X-ray intensity scans across low-angle synchrotron $\mathrm{x}$-ray diffraction patterns from the corneas of day 8 (D8) to 14 wild-type and lumicannull mice. The peak in the region of 0.013 to $0.018 \mathrm{~nm}^{-1}$ represents the collagen interfibrillar $\mathrm{x}$-ray reflection that arises from the regularly arranged collagen fibrils that constitute the stromal extracellular matrix.

\section{Discussion}

In vivo examinations of neonatal corneal development in the mouse have disclosed that the stroma undergoes a critical period of swelling and thinning around the time of eye opening in the day 8 to 14 interval. ${ }^{16}$ We performed a series of synchrotron x-ray diffraction experiments on groups of 10 or 12 normal mouse corneas obtained every two days during this developmental period to investigate the internal fine structure of the stroma and to chart any matrix alterations. Data revealed that in normal mice, the average collagen fibril spacing remained relatively unchanged between days 8 and 10 (Table 1). It then decreased around the time of eye opening (days 1012), so that a progressively more closely packed collagenous matrix was formed by day 14. Despite differences in tissue preservation and data acquisition/analysis, collagen fibrils in the neonatal mouse cornea throughout the day 8-14 time frame were more widely spaced, on average, than collagen fibrils in the corneas of more mature (i.e., 2- to 6-month-old) mice. $^{17,19}$
Confocal microscopy has found that, in vivo, stromal thickness in normal mice transiently peaks at day 12 , only to decrease by day 14 before resuming slow continued growth to adult thickness. ${ }^{16}$ The present data have not indicated an increase in average collagen interfibrillar spacing at day 12, leading us to conclude that stromal thickening at this time cannot be ascribed to homogeneous moving apart of collagen fibrils. (A preliminary report at the ARVO 2003 annual meeting presented initial evidence for elevated collagen fibril spacing at day 12 in the normal mouse stroma, but this is not substantiated by the current, more extensive data.) An alternative explanation for the transient day 12 stromal thickening might be the temporary presence of regions of the corneal stroma that are devoid of regularly arranged collagen fibrils, the so-called collagen-free lakes that have been evoked by Benedek ${ }^{23}$ among others. As alluded to earlier, $\mathrm{x}$-rays scattered by a corneal matrix of collagen fibrils with some spatial regularity will interfere to form a recordable interfibrillar $\mathrm{x}$-ray reflection. Through analysis, this yields a value for the average center-to- 
TABLE 1. Average Structural Parameters for $\mathrm{Lum}^{+/+}$and Lum ${ }^{-/-}$Mouse Corneas

\begin{tabular}{|c|c|c|c|c|c|c|}
\hline \multirow[b]{2}{*}{ Day } & \multicolumn{2}{|c|}{$\begin{array}{c}\text { Fibril } \\
\text { Spacing, } \mathbf{n m}\end{array}$} & \multicolumn{2}{|c|}{$\begin{array}{c}\text { Fibril } \\
\text { Diameter, nm }\end{array}$} & \multicolumn{2}{|c|}{$\begin{array}{c}\text { Coherence } \\
\text { Distance, } \mathbf{n m}\end{array}$} \\
\hline & $\operatorname{Lum}^{+/+}$ & $\mathbf{L u m}^{-/-}$ & $\operatorname{Lum}^{+/+}$ & $\mathbf{L u m}^{-/-}$ & $\mathbf{L u m}^{+/+}$ & $\mathbf{L u m}^{-/-}$ \\
\hline 8 & $65.4 \pm 3.6$ & $\begin{array}{l}75.6 \pm 3.8 \\
P<0.001\end{array}$ & $31.0 \pm 1.3$ & $\begin{array}{l}29.1 \pm 1.0 \\
P=0.005^{*}\end{array}$ & $247 \pm 23$ & $\mathrm{~N} / \mathrm{O}$ \\
\hline 10 & $64.6 \pm 3.4$ & $\begin{array}{l}69.2 \pm 3.4 \\
P=0.005\end{array}$ & $31.3 \pm 0.8$ & $\begin{array}{l}30.4 \pm 1.3 \\
P=0.056\end{array}$ & $239 \pm 14$ & $\begin{array}{c}197 \pm 15 \\
P<0.001\end{array}$ \\
\hline 12 & $63.0 \pm 3.6$ & $\begin{array}{l}68.4 \pm 3.2 \\
P<0.001\end{array}$ & $32.5 \pm 1.2$ & $\begin{array}{l}30.2 \pm 0.8 \\
P<0.001\end{array}$ & $227 \pm 16$ & $\begin{array}{r}181 \pm 10 \\
P<0.001\end{array}$ \\
\hline 14 & $61.4 \pm 3.7$ & $\begin{array}{l}63.8 \pm 3.1 \\
P=0.104\end{array}$ & $32.3 \pm 1.3$ & $\begin{array}{l}30.8 \pm 1.4 \\
P=0.015\end{array}$ & $227 \pm 14$ & $\begin{array}{l}199 \pm 14 \\
P<0.001\end{array}$ \\
\hline
\end{tabular}

\footnotetext{
All values are mean $\pm \mathrm{SD}$.

Comparison of neonatal murine Lum ${ }^{+/+}$and $\mathrm{Lum}^{-/-}$corneal collagen interfibrillar Bragg spacing, collagen fibril diameter, and level of fibrillar organization defined as the coherence distance, with higher numbers indicative of more local order in the stromal matrix. Collagen interfibrillar spacing is significantly higher in the lumican null corneal stroma at days 8,10 , and 12 , whereas fibril diameter is marginally reduced in the lumican null corneas almost throughout.

N/O indicates data not obtainable because of marked lack of local order in fibrillar array.

* Nonparametric testing because of nonnormal data distribution.
}

center collagen interfibrillar spacing. Regions of the cornea with little or no regularly arranged collagen, on the other hand, do not contribute sufficiently to the interfibrillar reflection; thus, the presence of lakes would remain unrecognized in the current analysis. It should be borne in mind that the lakes as envisaged here may constitute cellular components of the stroma. Therefore, the transient day 12 increase in stromal thickness can be reconciled with the current findings if it were caused by a passing increase in the overall keratocyte cell volume. Consequently, our interpretation of the data favors a mechanism of temporary stromal thickening in which expansion and contraction of stromal lakes, cellular or not, occur at approximately day 12 rather than a process involving a widespread, homogeneous adjustment of the collagen fibrillar array.

Unlike normal mouse cornea, the corneas of lumican-null mutants do not become thicker at neonatal day $12 .^{16}$ The x-ray data indicated that at days 8, 10, and 12, lumican-null corneas exhibited a significant increase in average collagen fibril spacing compared with their age-matched, wild-type counterparts (Table 1). This was particularly noticeable at day 8. By day 14, the average fibril spacing in the corneas of mutant mice was marginally higher than the corresponding wild-type value, but not significantly so. A previous investigation ${ }^{17}$ shows that the corneas of mature (2- to 6-month-old) lumican-deficient mice from the same colony have collagen fibrils that tend to be more widely spaced than collagen fibrils in the corneas of agematched wild-type littermates by an average of $2 \mathrm{~nm}$. Taken with the current findings, a picture emerges of a neonatal mouse cornea before eye opening that has a loosely packed stromal matrix in the absence of lumican. After eye opening, this difference recedes so that by day 14 the marginally elevated interfibrillar spacing in lumican-null corneas is not statistically significant (Table 1). As the tissue gains maturity, the evidence is that the fibrillar array becomes more compact in wild-type and lumican-null corneas alike, with x-ray diffraction data suggesting that a small average elevation in fibril spacing in the lumican-null cornea of approximately $2 \mathrm{~nm}$ persists. In some respects the finding of a marginally more loosely packed fibrillar stroma in the absence of a molecule, lumican, which ordinarily contains hydrophilic keratan sulfate side chains, is counterintuitive, but we point out that keratan sulfate tends to be less highly sulfated in the mouse cornea than in that of other species. ${ }^{24}$ Additionally, perhaps the hydrophilic nature of its stroma in the lumican-null situation is maintained by other matrix components.
In the normal mouse cornea, the average collagen fibril diameter during the day 8 to 14 neonatal period lies in the 31-nm to 32.5-nm range (Table 1), the same as for 4-month-old mouse corneas that were examined by synchrotron $\mathrm{x}$-ray diffraction after chemical fixation. ${ }^{18}$ (Aldehyde fixation causes shrinkage of corneal tissue, ${ }^{25}$ with the average collagen fibril diameters in hydrated, unfixed normal adult mouse corneas closer to $35 \mathrm{~nm} .{ }^{17,19}$ ) At all time points investigated here, the average collagen fibril diameter in lumican-null corneas was lower (29.1-30.8 nm) than the average collagen fibril diameter in normal corneas, with the difference significant or close to significant (Table 1). We emphasize that this is a representative average value from $\mathrm{x}$-ray diffraction. Previous electron microscopic studies have found unusually large, fused collagen fibrils in the lumican-null cornea. ${ }^{26}$ These fused fibrils are mostly in the posterior stroma, but because they are present only at low levels, they will not contribute appreciably to the x-ray diffraction pattern. Previous experiments have shown that lumican, extracted from bovine cornea by salt or guanidine treatment, is active in inhibiting the in vitro fibrillogenesis of purified collagen monomers, also obtained from ox cornea. ${ }^{27}$ In those experiments, fibrils that were formed in the absence of lumican achieved significantly higher final diameters. Ostensibly, this is at odds with the data presented here because the average fibril diameter in our lumican-deficient mice is, throughout the developmental period studied, lower than normal. The discrepancy between the findings of the in vitro experiments with bovine corneal extracts ${ }^{27}$ and the transgenic mouse work presented here is difficult to pinpoint, but perhaps it is attributed to a host of reasons. Maybe other stromal proteoglycans or matrix components compensate for the lack of lumican in our mouse model. After all, decorin proteoglycan and decorin core protein also have the ability to regulate collagen fibril diameter. ${ }^{27}$ We should also consider that collagen type $\mathrm{V}$ has been identified as a regulator of fibril diameter in the hybrid type I/ $\mathrm{V}$ fibrils that are found in cornea. ${ }^{28}$ Thus, it is possible that a differential influence of collagen type $\mathrm{V}$ might have contributed to the disparate results in the two systems (the in vitro bovine work used an estimated mixture of $91 \%$ collagen type I and $9 \%$ collagen type $\mathrm{V}^{27}$ ). We can also speculate that some type of fibril fusion might have taken place in the in vitro bovine fibrillogenesis experiments. ${ }^{27}$

Coherence distance measurements for the wild-type corneas between days 8 and 14 are not markedly different (Table 1 ), indicating that the level of local order in the normal mouse 
stroma does not change appreciably between days $8,10,12$, and 14. Put another way, we can conclude that the corneal stroma of the normal mouse is already structurally well organized before eye opening, and that its becoming organized is not a consequence of the stromal condensation that occurs after eye opening. The corneal stroma of the lumican-null mouse, on the other hand, is, at all times studied, less wellorganized than the corneal stroma of the normal mouse. At day 8 , in particular, the stromal matrix of the lumican-null mouse was so disorganized that it did not enable us to make reliable measurements of the coherence distance across all corneas in that group. Stromal disorganization in lumican-deficient mice persists into adulthood. ${ }^{17}$ It is also a feature of mature keratocan-deficient corneas, ${ }^{18}$ though not, to any great extent, mature mimecan-deficient corneas. ${ }^{19}$

The current findings point to a key role for lumican in the establishment of a properly organized stromal matrix in the neonatal developmental phase, presumably through its interaction with collagen and other matrix components, and suggest that the structural defects seen in adult lumican-null corneas are not structural problems that arise de novo with age, but stem from early postnatal events.

\section{Acknowledgments}

The authors thank Gunter Grossmann for help at the Synchotron Radiation Source (Daresbury Laboratory, Cheshire, UK) and Rob Young for useful discussions.

\section{References}

1. Maurice DM. The structure and transparency of the cornea. J Physiol. 1957;136:263-286.

2. Anseth A. Glycosaminoglycans in corneal regeneration. Exp Eye Res. 1961;1:122-127.

3. Hassell JR, Cintron C, Kublin C, Newsome DA. Proteoglycan changes during restoration of transparency in corneal scars. Arch Biochem Biophys. 1983;222:362-369.

4. Nakazawa K, Hassell JR, Hascall VC, Lohmander LS, Newsome DA, Krachmer J. Defective processing of keratan sulfate in macular corneal dystrophy. J Biol Chem. 1984;259:22:13751-13757.

5. Yang CJ, SundarRaj N, Thonar J-Ma, Klintworth GK. Immunohistochemical evidence of heterogeneity in macular corneal dystrophy. Am J Ophthalmol. 1988;106:65-71.

6. Quantock AJ, Meek KM, Ridgway AEA, Bron AJ, Thonar EJ-MA. Macular corneal dystrophy: reduction in both corneal thickness and collagen interfibrillar spacing. Curr Eye Res. 1990;9:4:393398.

7. Anseth A. Glycosaminoglycans in the developing corneal stroma Exp Eye Res. 1961;1:116-121.

8. Cornuet PK, Blochberger TC, Hassell JR. Molecular polymorphism of lumican during corneal development. Invest Ophthalmol Vis Sci. 1994;35:870 - 877.

9. Connon CJ, Meek KM, Kinoshita S, Quantock AJ. Spatial and temporal alterations in the collagen fibrillar array during the onset of transparency in the avian cornea. Exp Eye Res. 2004;78:909915.
10. Blochberger TC, Vergnes JP, Hempel J, Hassell JR. cDNA to chick lumican (corneal keratan sulfate proteoglycan) reveals homology to the small interstitial proteoglycan gene family and expression in muscle and intestine. J Biol Chem. 1992;267:1:347-352.

11. Corpuz LM, Funderburgh JL, Funderburgh ML, Bottomley GS, Prakash S, Conrad GW. Molecular cloning and tissue distribution of keratocan: bovine corneal keratan sulfate proteoglycan 37A. J Biol Chem. 1996;271:16:9759-9763.

12. Funderburgh JL, Corpuz LM, Roth MR, Funderburgh ML, Tasheva ES, Conrad GW. Mimecan, the $25-\mathrm{kDa}$ corneal keratan sulfate proteoglycan, is a product of the gene producing osteoglycin. J Biol Chem. 1997;272:44:28089-28095.

13. Liu CY, Birk DE, Hassell JR, Kane B, Kao WWY. Keratocan-deficient mice display alterations in corneal structure. J Biol Chem. 2003;278:21672-21677.

14. Tasheva ES, Koester A, Paulson AQ, et al. Mimecan/osteoglycindeficient mice have collagen fibril abnormalities. Mol Vis. 2002;8: 407-415.

15. Chakravarti S, Magnuson T, Lass JH, Jepsen KJ, LaMantia C, Carroll $\mathrm{H}$. Lumican regulates collagen fibril assembly: skin fragility and corneal opacity in the absence of lumican. J Cell Biol. 1998;141: 1277-1286.

16. Song J, Lee Y-G, Houston J, et al. Neonatal corneal stromal development in the normal and lumican-deficient mouse. Invest Ophthalmol Vis Sci. 2003;44:548-557.

17. Quantock AJ, Meek KM, Chakravarti S. An x-ray diffraction investigation of corneal structure in lumican-deficient mice. Invest $O p h$ thalmol Vis Sci. 2001;42:1750-1756.

18. Meek KM, Quantock AJ, Boote C, Liu CY, Kao WWY. An X-ray investigation of corneal structure in keratocan-deficient mice. $\mathrm{Ma}$ trix Biol. 2003;22:467-475.

19. Beecher N, Carlson C, Allen BR, et al. An x-ray diffraction study of corneal structure in mimecan-deficient mice. Invest Ophthalmol Vis Sci. 2005;46:4046-4049.

20. Meek KM, Quantock AJ. The use of $x$-ray scattering techniques to determine corneal ultrastructure. Prog Ret Eye Res. 2001;20:95137.

21. Stokes AR. The theory of x-ray fibre diagrams. Prog Biophys. 1955;5:5-167.

22. Regini JW, Elliott GF, Hodson SA. The ordering of corneal collagen fibrils with increasing ionic strength. J Mol Biol. 2004;336:179186.

23. Benedek GB. Theory of transparency of the eye. Applied Optics. 1971;10:3:459-473.

24. Young RD, Tudor D, Hayes AJ, et al. Atypical composition and ultrastructure of proteoglycans in the mouse corneal stroma. Invest Ophthalmol Vis Sci. 2005;1973-1978.

25. Fullwood NJ, Meek KM. A synchrotron x-ray study of the changes occurring in the corneal stroma during processing for electron microscopy. J Microsc. 1992;169:53-60.

26. Chakravarti S, Petroll WM, Hassell JR, et al. Corneal opacity in lumican-null mice: defects in collagen fibril structure and packing in the posterior stroma. Invest Ophthalmol Vis Sci. 2000;41:11: 3365-3373.

27. Rada JA, Cornuet PK, Hassell JR. Regulation of corneal collagen fibrillogenesis in vitro by corneal proteoglycan (lumican and decorin) core proteins. Exp Eye Res. 1993;56:635-648.

28. Birk DE, Fitch JM, Babiarz JP, Doane KJ, Linsenmayer TF. Collagen fibrillogenesis in vitro-interaction of type-I and type-V collagen regulates fibril diameter. J Cell Sci. 1990;95:649-657. 